\title{
Strong Convergence Theorems of Common Fixed Points for a Family of Quasi- $\phi$-Nonexpansive Mappings
}

\author{
Xiaolong Qin, ${ }^{1}$ Yeol Je Cho, ${ }^{2}$ Sun Young Cho, ${ }^{3}$ \\ and Shin Min Kang ${ }^{4}$ \\ ${ }^{1}$ Department of Mathematics, Hangzhou Normal University, Hangzhou 310036, China \\ ${ }^{2}$ Department of Mathematics Education and the RINS, Gyeongsang National University, \\ Jinju 660-701, South Korea \\ ${ }^{3}$ Department of Mathematics, Gyeongsang National University, Jinju 660-701, South Korea \\ ${ }^{4}$ Department of Mathematics and the RINS, Gyeongsang National University, \\ Jinju 660-701, South Korea
}

Correspondence should be addressed to Shin Min Kang, smkang@gnu.ac.kr

Received 31 August 2009; Accepted 19 November 2009

Academic Editor: Tomonari Suzuki

Copyright (C) 2010 Xiaolong Qin et al. This is an open access article distributed under the Creative Commons Attribution License, which permits unrestricted use, distribution, and reproduction in any medium, provided the original work is properly cited.

We consider a modified Halpern type iterative algorithm for a family of quasi- $\phi$-nonexpansive mappings in the framework of Banach spaces. Strong convergence theorems of the purposed iterative algorithms are established.

\section{Introduction}

Let $E$ be a Banach space, $C$ a nonempty closed and convex subset of $E$, and $T: C \rightarrow C$ a nonlinear mapping. Recall that $T$ is nonexpansive if

$$
\|T x-T y\| \leq\|x-y\|, \quad \forall x, y \in C .
$$

A point $x \in C$ is a fixed point of $T$ provided $T x=x$. Denote by $F(T)$ the set of fixed points of $T$, that is, $F(T)=\{x \in C: T x=x\}$. 
One classical way to study nonexpansive mappings is to use contractions to approximate a nonexpansive mapping; see $([1,2])$. More precisely, take $t \in(0,1)$ and define a contraction $T_{t}: C \rightarrow C$ by

$$
T_{t} x=t u+(1-t) T x, \quad \forall x \in C,
$$

where $u \in C$ is a fixed element. Banach Contraction Mapping Principle guarantees that $T_{t}$ has a unique fixed point $x_{t}$ in $C$. It is unclear, in general, what the behavior of $x_{t}$ is as $t \rightarrow 0$ even if $T$ has a fixed point. However, in the case of $T$ having a fixed point, Browder [1] proved the following well-known strong convergence theorem.

Theorem B. Let $C$ be a bounded closed convex subset of a Hilbert space $H$ and $T$ a nonexpansive mapping on $C$. Fix $u \in C$ and define $z_{t} \in C$ as $z_{t}=t u+(1-t) T z_{t}$ for any $t \in(0,1)$. Then $\left\{z_{t}\right\}$ converges strongly to an element of $F(T)$ nearest to $u$.

Motivated by Theorem B, Halpern [3] considered the following explicit iteration:

$$
x_{0} \in C, \quad x_{n+1}=\alpha_{n} u+\left(1-\alpha_{n}\right) T x_{n}, \quad \forall n \geq 0,
$$

and obtained the following theorem.

Theorem H. Let $C$ be a bounded closed convex subset of a Hilbert space $H$ and $T$ a nonexpansive mapping on $C$. Define a real sequence $\left\{\alpha_{n}\right\}$ in $[0,1]$ by $\alpha_{n}=n^{-\theta}, 0<\theta<1$. Then the sequence $\left\{x_{n}\right\}$ defined by (1.3) converges strongly to the element of $F(T)$ nearest to $u$.

In [4], Lions improved the result of Halpern [3], still in Hilbert spaces, by proving the strong convergence of $\left\{x_{n}\right\}$ to a fixed point of $T$ provided that the control sequence $\left\{\alpha_{n}\right\}$ satisfies the following conditions:

(C1) $\lim _{n \rightarrow \infty} \alpha_{n}=0$;

(C2) $\sum_{n=1}^{\infty} \alpha_{n}=\infty$;

(C3) $\lim _{n \rightarrow \infty}\left(\left(\alpha_{n+1}-\alpha_{n}\right) / \alpha_{n+1}^{2}\right)=0$.

It was observed that both the Halpern's and Lion's conditions on the real sequence $\left\{\alpha_{n}\right\}$ excluded the canonical choice $\left\{\alpha_{n}\right\}=1 /(n+1)$. This was overcome by Wittmann [5], who proved, still in Hilbert spaces, the strong convergence of $\left\{x_{n}\right\}$ to a fixed point of $T$ if $\left\{\alpha_{n}\right\}$ satisfies the following conditions:

(C1) $\lim _{n \rightarrow \infty} \alpha_{n}=0$;

(C2) $\sum_{n=1}^{\infty} \alpha_{n}=\infty$;

(C4) $\sum_{n=1}^{\infty}\left|\alpha_{n+1}-\alpha_{n}\right|<\infty$.

In [6], Shioji and Takahashi extended Wittmann's results to the setting of Banach spaces under the assumptions (C1), (C2), and (C4) imposed on the control sequences $\left\{\alpha_{n}\right\}$. In [7], Xu remarked that the conditions (C1) and (C2) are necessary for the strong convergence of the iterative sequence defined in (1.3) for all nonexpansive self-mappings. It is well known that the iterative algorithm (1.3) is widely believed to have slow convergence because 
the restriction of condition $(\mathrm{C} 2)$. Thus, to improve the rate of convergence of the iterative process (1.3), one cannot rely only on the process itself.

Recently, hybrid projection algorithms have been studied for the fixed point problems of nonlinear mappings by many authors; see, for example, [8-24]. In 2006, Martinez-Yanes and $\mathrm{Xu}[10]$ proposed the following modification of the Halpern iteration for a single nonexpansive mapping $T$ in a Hilbert space. To be more precise, they proved the following theorem.

Theorem MYX. Let $H$ be a real Hilbert space, $C$ a closed convex subset of $H$, and $T: C \rightarrow C$ a nonexpansive mapping such that $F(T) \neq \emptyset$. Assume that $\left\{\alpha_{n}\right\} \subset(0,1)$ is such that $\lim _{n \rightarrow \infty} \alpha_{n}=0$. Then the sequence $\left\{x_{n}\right\}$ defined by

$$
\begin{gathered}
x_{0} \in C \text { chosen arbitrarily, } \\
y_{n}=\alpha_{n} x_{0}+\left(1-\alpha_{n}\right) T x_{n}, \\
C_{n}=\left\{z \in C:\left\|y_{n}-z\right\|^{2} \leq\left\|x_{n}-z\right\|^{2}+\alpha_{n}\left(\left\|x_{0}\right\|^{2}+2\left\langle x_{n}-x_{0}, z\right\rangle\right)\right\}, \\
Q_{n}=\left\{z \in C:\left\langle x_{0}-x_{n}, x_{n}-z\right\rangle \geq 0\right\}, \\
x_{n+1}=P_{C_{n} \cap Q_{n}} x_{0}, \quad \forall n \geq 0,
\end{gathered}
$$

converges strongly to $P_{F(T)} x_{0}$.

Very recently, Qin and Su [17] improved the result of Martinez-Yanes and Xu [10] from Hilbert spaces to Banach spaces. To be more precise, they proved the following theorem.

Theorem QS. Let E be a uniformly convex and uniformly smooth Banach space, $C$ a nonempty closed convex subset of $E$, and $T: C \rightarrow C$ a relatively nonexpansive mapping. Assume that $\left\{\alpha_{n}\right\}$ is a sequence in $(0,1)$ such that $\lim _{n \rightarrow \infty} \alpha_{n}=0$. Define a sequence $\left\{x_{n}\right\}$ in $C$ by the following algorithm:

$$
\begin{gathered}
x_{0} \in C \quad \text { chosen arbitrarily, } \\
y_{n}=J^{-1}\left(\alpha_{n} J x_{0}+\left(1-\alpha_{n}\right) J T x_{n}\right), \\
C_{n}=\left\{v \in C: \phi\left(v, y_{n}\right) \leq \alpha_{n} \phi\left(v, x_{0}\right)+\left(1-\alpha_{n}\right) \phi\left(v, x_{n}\right)\right\}, \\
Q_{n}=\left\{v \in C:\left\langle J x_{0}-J x_{n}, x_{n}-v\right\rangle \geq 0\right\}, \\
x_{n+1}=\prod_{C_{n} \cap Q_{n}} x_{0}, \quad \forall n \geq 0,
\end{gathered}
$$

where $J$ is the single-valued duality mapping on E. If $F(T)$ is nonempty, then $\left\{x_{n}\right\}$ converges to $\Pi_{F(T)} x_{0}$.

In this paper, motivated by Kimura and Takahashi [8], Martinez-Yanes and Xu [10], Qin and Su [17], and Qin et al. [19], we consider a hybrid projection algorithm to modify the iterative process (1.3) to have strong convergence under condition (C1) only for a family of closed quasi- $\phi$-nonexpansive mappings. 


\section{Preliminaries}

Let $E$ be a Banach space with the dual space $E^{*}$. We denote by $J$ the normalized duality mapping from $E$ to $2^{E^{*}}$ defined by

$$
J x=\left\{f^{*} \in E^{*}:\left\langle x, f^{*}\right\rangle=\|x\|^{2}=\left\|f^{*}\right\|^{2}\right\}, \quad \forall x \in E,
$$

where $\langle\cdot, \cdot\rangle$ denotes the generalized duality pairing. It is well known that, if $E^{*}$ is strictly convex, then $J$ is single-valued and, if $E^{*}$ is uniformly convex, then $J$ is uniformly continuous on bounded subsets of $E$.

We know that, if $C$ is a nonempty closed convex subset of a Hilbert space $H$ and $P_{C}: H \rightarrow C$ is the metric projection of $H$ onto $C$, then $P_{C}$ is nonexpansive. This fact actually characterizes Hilbert spaces and, consequently, it is not available in more general Banach spaces. In this connection, Alber [25] recently introduced a generalized projection operator $\Pi_{C}$ in a Banach space $E$, which is an analogue of the metric projection in Hilbert spaces.

A Banach space $E$ is said to be strictly convex if $\|(x+y) / 2\|<1$ for all $x, y \in E$ with $\|x\|=\|y\|=1$ and $x \neq y$. The space $E$ is said to be uniformly convex if $\lim _{n \rightarrow \infty}\left\|x_{n}-y_{n}\right\|=0$ for any two sequences $\left\{x_{n}\right\}$ and $\left\{y_{n}\right\}$ in $E$ such that $\left\|x_{n}\right\|=\left\|y_{n}\right\|=1$ and $\lim _{n \rightarrow \infty}\left\|\left(x_{n}+y_{n}\right) / 2\right\|=1$. Let $U=\{x \in E:\|x\|=1\}$ be the unit sphere of $E$. Then the space $E$ is said to be smooth if

$$
\lim _{t \rightarrow 0} \frac{\|x+t y\|-\|x\|}{t}
$$

exists for each $x, y \in U$. It is also said to be uniformly smooth if the limit is attained uniformly for $x, y \in E$. It is well known that, if $E$ is uniformly smooth, then $J$ is uniformly norm-to-norm continuous on each bounded subset of $E$.

In a smooth Banach space $E$, we consider the functional defined by

$$
\phi(x, y)=\|x\|^{2}-2\langle x, J y\rangle+\|y\|^{2}, \quad \forall x, y \in E .
$$

Observe that, in a Hilbert space $H,(2.3)$ reduces to $\phi(x, y)=\|x-y\|^{2}$ for all $x, y \in H$. The generalized projection $\Pi_{C}: E \rightarrow C$ is a mapping that assigns to an arbitrary point $x \in E$ the minimum point of the functional $\phi(x, y)$, that is, $\Pi_{C} x=\bar{x}$, where $\bar{x}$ is the solution to the minimization problem:

$$
\phi(\bar{x}, x)=\min _{y \in C} \phi(y, x)
$$

The existence and uniqueness of the operator $\Pi_{C}$ follows from some properties of the functional $\phi(x, y)$ and the strict monotonicity of the mapping $J$ (see, e.g., [25-28]). In Hilbert spaces, $\Pi_{C}=P_{C}$. It is obvious from the definition of the function $\phi$ that

$$
(\|y\|-\|x\|)^{2} \leq \phi(y, x) \leq(\|y\|+\|x\|)^{2}, \quad \forall x, y \in E .
$$

Remark 2.1. If $E$ is a reflexive, strictly convex, and smooth Banach space, then, for any $x, y \in E$, $\phi(x, y)=0$ if and only if $x=y$. In fact, it is sufficient to show that, if $\phi(x, y)=0$, then $x=y$. 
From (2.5), we have $\|x\|=\|y\|$. This implies $\langle x, J y\rangle=\|x\|^{2}=\|J y\|^{2}$. From the definition of $J$, one has $J x=J y$. Therefore, we have $x=y$ (see $[27,29]$ for more details).

Let $C$ be a nonempty closed and convex subset of $E$ and $T$ a mapping from $C$ into itself. A point $p \in C$ is said to be an asymptotic fixed point of $T$ ([30]) if $C$ contains a sequence $\left\{x_{n}\right\}$ which converges weakly to $p$ such that $\lim _{n \rightarrow \infty}\left\|x_{n}-T x_{n}\right\|=0$. The set of asymptotic fixed points of $T$ will be denoted by $\widetilde{F(T)}$. A mapping $T$ from $C$ into itself is said to be relatively nonexpansive $([27,31,32])$ if $\widetilde{F(T)}=F(T)$ and $\phi(p, T x) \leq \phi(p, x)$ for all $x \in C$ and $p \in F(T)$. The asymptotic behavior of a relatively nonexpansive mapping was studied by some authors $([27,31,32])$.

A mapping $T: C \rightarrow C$ is said to be $\phi$-nonexpansive $([18,19,24])$ if $\phi(T x, T y) \leq \phi(x, y)$ for all $x, y \in C$. The mapping $T$ is said to be quasi- $\phi$-nonexpansive $([18,19,24])$ if $F(T) \neq \emptyset$ and $\phi(p, T x) \leq \phi(p, x)$ for all $x \in C$ and $p \in F(T)$.

Remark 2.2. The class of quasi- $\phi$-nonexpansive mappings is more general than the class of relatively nonexpansive mappings, which requires the strong restriction: $F(T)=\widetilde{F(T)}$.

In order to prove our main results, we need the following lemmas.

Lemma 2.3 (see [28]). Let E be a uniformly convex and smooth Banach space and $\left\{x_{n}\right\},\left\{y_{n}\right\}$ two sequences of $E$. If $\phi\left(x_{n}, y_{n}\right) \rightarrow 0$ and either $\left\{x_{n}\right\}$ or $\left\{y_{n}\right\}$ is bounded, then $x_{n}-y_{n} \rightarrow 0$.

Lemma 2.4 (see $[25,28]$ ). Let $C$ be a nonempty closed convex subset of a smooth Banach space $E$ and $x \in E$. Then $x_{0}=\Pi_{C} x \in C$ if and only if

$$
\left\langle x_{0}-y, J x-J x_{0}\right\rangle \geq 0, \quad \forall y \in C
$$

Lemma 2.5 (see $[25,28]$ ). Let $E$ be a reflexive, strictly convex, and smooth Banach space, $C$ a nonempty closed convex subset of $E$ and $x \in E$. Then

$$
\phi\left(y, \Pi_{C} x\right)+\phi\left(\Pi_{C} x, x\right) \leq \phi(y, x), \quad \forall y \in C
$$

Lemma 2.6 (see $[7,18]$ ). Let E be a uniformly convex and smooth Banach space, $C$ a nonempty, closed, and convex subset of $E$ and $T$ a closed quasi- $\phi$-nonexpansive mapping from $C$ into itself. Then $F(T)$ is a closed and convex subset of $C$.

\section{Main Results}

From now on, we use $I$ to denote an index set. Now, we are in a position to prove our main results.

Theorem 3.1. Let $C$ be a nonempty closed and convex subset of a uniformly convex and uniformly smooth Banach space $E$ and $\left\{T_{i}\right\}_{i \in I}: C \rightarrow C$ a family of closed quasi- $\phi$-nonexpansive mappings 
such that $F=\bigcap_{i \in I} F\left(T_{i}\right) \neq \emptyset$. Let $\left\{\alpha_{n}\right\}$ be a real sequence in $(0,1)$ such that $\lim _{n \rightarrow \infty} \alpha_{n}=0$. Define a sequence $\left\{x_{n}\right\}$ in $C$ in the following manner:

$$
\begin{gathered}
x_{0} \in C \text { chosen arbitrarily, } \\
y_{(n, i)}=J^{-1}\left[\alpha_{n} J x_{0}+\left(1-\alpha_{n}\right) J T_{i} x_{n}\right], \\
C_{(n, i)}=\left\{z \in C: \phi\left(z, y_{(n, i)}\right) \leq \alpha_{n} \phi\left(z, x_{0}\right)+\left(1-\alpha_{n}\right) \phi\left(z, x_{n}\right)\right\}, \\
C_{n}=\bigcap_{i \in I} C_{(n, i)}, \\
Q_{0}=C, \\
Q_{n}=\left\{z \in Q_{n-1}:\left\langle x_{n}-z, J x_{0}-J x_{n}\right\rangle \geq 0\right\}, \\
x_{n+1}=\prod_{C_{n} \cap Q_{n}} x_{0}, \quad \forall n \geq 0,
\end{gathered}
$$

then the sequence $\left\{x_{n}\right\}$ defined by (3.1) converges strongly to $\Pi_{F} x_{0}$.

Proof. We first show that $C_{n}$ and $Q_{n}$ are closed and convex for each $n \geq 0$. From the definitions of $C_{n}$ and $Q_{n}$, it is obvious that $C_{n}$ is closed and $Q_{n}$ is closed and convex for each $n \geq 0$. We, therefore, only show that $C_{n}$ is convex for each $n \geq 0$. Indeed, note that

$$
\phi\left(z, y_{(n, i)}\right) \leq \alpha_{n} \phi\left(z, x_{0}\right)+\left(1-\alpha_{n}\right) \phi\left(z, x_{n}\right)
$$

is equivalent to

$$
2 \alpha_{n}\left\langle z, J x_{0}\right\rangle+2\left(1-\alpha_{n}\right)\left\langle z, J x_{n}\right\rangle-2\left\langle z, J y_{n, i}\right\rangle \leq \alpha_{n}\left\|x_{0}\right\|^{2}+\left(1-\alpha_{n}\right)\left\|x_{n}\right\|^{2}-\left\|y_{(n, i)}\right\|^{2} .
$$

This shows that $C_{(n, i)}$ is closed and convex for each $n \geq 0$ and $i \in I$. Therefore, we obtain that $C_{n}=\bigcap_{i \in I} C_{(n, i)}$ is convex for each $n \geq 0$.

Next, we show that $F \subset C_{n}$ for all $n \geq 0$. For each $w \in F$ and $i \in I$, we have

$$
\begin{aligned}
\phi\left(w, y_{(n, i)}\right) & =\phi\left(w, J^{-1}\left[\alpha_{n} J x_{0}+\left(1-\alpha_{n}\right) J T_{i} x_{n}\right]\right) \\
& =\|w\|^{2}-2\left\langle w, \alpha_{n} J x_{0}+\left(1-\alpha_{n}\right) J T_{i} x_{n}\right\rangle+\left\|\alpha_{n} J x_{0}+\left(1-\alpha_{n}\right) J T_{i} x_{n}\right\|^{2} \\
& \leq\|w\|^{2}-2 \alpha_{n}\left\langle w, J x_{0}\right\rangle+2\left(1-\alpha_{n}\right)\left\langle w, J T_{i} x_{n}\right\rangle+\alpha_{n}\left\|x_{0}\right\|^{2}+\left(1-\alpha_{n}\right)\left\|T_{i} x_{n}\right\|^{2} \\
& \leq \alpha_{n} \phi\left(w, x_{0}\right)+\left(1-\alpha_{n}\right) \phi\left(w, T_{i} x_{n}\right) \\
& \leq \alpha_{n} \phi\left(w, x_{0}\right)+\left(1-\alpha_{n}\right) \phi\left(w, x_{n}\right),
\end{aligned}
$$

which yields that $w \in C_{(n, i)}$ for all $n \geq 0$ and $i \in$ I. It follows that $w \in C_{n}=\bigcap_{i \in I} C_{(n, i)}$. This proves that $F \subset C_{n}$ for all $n \geq 0$. 
Next, we prove that $F \subset Q_{n}$ for all $n \geq 0$. We prove this by induction. For $n=0$, we have $F \subset C=Q_{0}$. Assume that $F \subset Q_{n-1}$ for some $n \geq 1$. Next, we show that $F \subset Q_{n}$ for the same $n$. Since $x_{n}$ is the projection of $x_{0}$ onto $C_{n-1} \cap Q_{n-1}$, we obtain that

$$
\left\langle x_{n}-z, J x_{0}-J x_{n}\right\rangle \geq 0, \quad \forall z \in C_{n-1} \cap Q_{n-1} .
$$

Since $F \subset C_{n-1} \cap Q_{n-1}$ by the induction assumption, (3.5) holds, in particular, for all $w \in F$. This together with the definition of $Q_{n}$ implies that $F \subset Q_{n}$ for all $n \geq 0$. Noticing that $x_{n+1}=$ $\Pi_{C_{n} \cap Q_{n}} x_{0} \in Q_{n}$ and $x_{n}=\Pi_{Q_{n}} x_{0}$, one has

$$
\phi\left(x_{n}, x_{0}\right) \leq \phi\left(x_{n+1}, x_{0}\right), \quad \forall n \geq 0 .
$$

We, therefore, obtain that $\left\{\phi\left(x_{n}, x_{0}\right)\right\}$ is nondecreasing. From Lemma 2.5, we see that

$$
\begin{aligned}
\phi\left(x_{n}, x_{0}\right) & =\phi\left(\Pi_{C_{n}} x_{0}, x_{0}\right) \\
& \leq \phi\left(w, x_{0}\right)-\phi\left(w, x_{n}\right) \\
& \leq \phi\left(w, x_{0}\right), \quad \forall w \in F \subset C_{n}, \forall n \geq 0 .
\end{aligned}
$$

This shows that $\left\{\phi\left(x_{n}, x_{0}\right)\right\}$ is bounded. It follows that the limit of $\left\{\phi\left(x_{n}, x_{0}\right)\right\}$ exists. By the construction of $Q_{n}$, we see that $Q_{m} \subset Q_{n}$ and $x_{m}=\Pi_{Q_{m}} x_{0} \in Q_{n}$ for any positive integer $m \geq n$. Notice that

$$
\begin{aligned}
\phi\left(x_{m}, x_{n}\right) & =\phi\left(x_{m}, \Pi_{C_{n}} x_{0}\right) \\
& \leq \phi\left(x_{m}, x_{0}\right)-\phi\left(\Pi_{C_{n}} x_{0}, x_{0}\right) \\
& =\phi\left(x_{m}, x_{0}\right)-\phi\left(x_{n}, x_{0}\right) .
\end{aligned}
$$

Taking the limit as $m, n \rightarrow \infty$ in (3.8), we get that $\phi\left(x_{m}, x_{n}\right) \rightarrow 0$. From Lemma 2.3, one has $x_{m}-x_{n} \rightarrow 0$ as $m, n \rightarrow \infty$. It follows that $\left\{x_{n}\right\}$ is a Cauchy sequence in $C$. Since $E$ is a Banach space and $C$ is closed and convex, we can assume that $x_{n} \rightarrow q \in C$ as $n \rightarrow \infty$.

Finally, we show that $q=\Pi_{F} x_{0}$. To end this, we first show $q \in F$. By taking $m=n+1$ in (3.8), we have

$$
\phi\left(x_{n+1}, x_{n}\right) \longrightarrow 0 \quad(n \longrightarrow \infty)
$$

From Lemma 2.3, we arrive at

$$
x_{n+1}-x_{n} \longrightarrow 0 \quad(n \longrightarrow \infty)
$$

Noticing that $x_{n+1} \in C_{n}$, we obtain

$$
\phi\left(x_{n+1}, y_{(n, i)}\right) \leq \alpha_{n} \phi\left(x_{n+1}, x_{0}\right)+\left(1-\alpha_{n}\right) \phi\left(x_{n+1}, x_{n}\right)
$$


It follows from the assumption on $\left\{\alpha_{n}\right\}$ and (3.9) that $\lim _{n \rightarrow \infty} \phi\left(x_{n+1}, y_{(n, i)}\right)=0$ for each $i \in I$. From Lemma 2.3, we obtain

$$
\lim _{n \rightarrow \infty}\left\|x_{n+1}-y_{(n, i)}\right\|=0, \quad \forall i \in I
$$

On the other hand, we have $\left\|J y_{(n, i)}-J T_{i} x_{n}\right\|=\alpha_{n}\left\|J x_{0}-J T_{i} x_{n}\right\|$. By the assumption on $\left\{\alpha_{n}\right\}$, we see that $\lim _{n \rightarrow \infty}\left\|J y_{(n, i)}-J T_{i} x_{n}\right\|=0$ for each $i \in I$. Since $J^{-1}$ is also uniformly norm-to-norm continuous on bounded sets, we obtain that

$$
\lim _{n \rightarrow \infty}\left\|y_{(n, i)}-T_{i} x_{n}\right\|=0
$$

On the other hand, we have

$$
\left\|x_{n}-T_{i} x_{n}\right\| \leq\left\|x_{n}-x_{n+1}\right\|+\left\|x_{n+1}-y_{(n, i)}\right\|+\left\|y_{(n, i)}-T_{i} x_{n}\right\|
$$

From (3.10)-(3.13), we obtain $\lim _{n \rightarrow \infty}\left\|T_{i} x_{n}-x_{n}\right\|=0$. From the closedness of $T_{i}$, we get $q \in F$. Finally, we show that $q=\Pi_{F} x_{0}$. From $x_{n}=\Pi_{C_{n}} x_{0}$, we see that

$$
\left\langle x_{n}-w, J x_{0}-J x_{n}\right\rangle \geq 0, \quad \forall w \in F \subset C_{n}
$$

Taking the limit as $n \rightarrow \infty$ in (3.15), we obtain that

$$
\left\langle q-w, J x_{0}-J q\right\rangle \geq 0, \quad \forall w \in F
$$

and hence $q=\Pi_{F} x_{0}$ by Lemma 2.4. This completes the proof.

Remark 3.2. Comparing the hybrid projection algorithm (3.1) in Theorem 3.1 with algorithm (1.5) in Theorem QS, we remark that the set $Q_{n}$ is constructed based on the set $Q_{n-1}$ instead of $C$ for each $n \geq 1$. We obtain that the sequence generated by the algorithm (3.1) is a Cauchy sequence. The proof is, therefore, different from the one presented in Qin and Su [17].

As a corollary of Theorem 3.1, for a single quasi- $\phi$-nonexpansive mapping, we have the following result immediately.

Corollary 3.3. Let $C$ be a nonempty, closed, and convex subset of a uniformly convex and uniformly smooth Banach space $E$ and $T: C \rightarrow C$ a closed quasi- $\phi$-nonexpansive mappings with a fixed point. 
Let $\left\{\alpha_{n}\right\}$ be a real sequence in $(0,1)$ such that $\lim _{n \rightarrow \infty} \alpha_{n}=0$. Define a sequence $\left\{x_{n}\right\}$ in $C$ in the following manner:

$$
\begin{gathered}
x_{0} \in C \quad \text { chosen arbitrarily, } \\
y_{n}=J^{-1}\left[\alpha_{n} J x_{0}+\left(1-\alpha_{n}\right) J T x_{n}\right], \\
C_{n}=\left\{z \in C: \phi\left(z, y_{n}\right) \leq \alpha_{n} \phi\left(z, x_{0}\right)+\left(1-\alpha_{n}\right) \phi\left(z, x_{n}\right)\right\}, \\
Q_{0}=C, \\
Q_{n}=\left\{z \in Q_{n-1}:\left\langle x_{n}-z, J x_{0}-J x_{n}\right\rangle \geq 0\right\}, \\
x_{n+1}=\prod_{C_{n} \cap Q_{n}} x_{0}, \quad \forall n \geq 0,
\end{gathered}
$$

then the sequence $\left\{x_{n}\right\}$ converges strongly to $\Pi_{F} x_{0}$.

Remark 3.4. Corollary 3.3 mainly improves Theorem 2.2 of Qin and Su [17] from the class of relatively nonexpansive mappings to the class of quasi- $\phi$-nonexpansive mappings, which relaxes the strong restriction: $\widetilde{F(T)}=F(T)$.

In the framework of Hilbert spaces, Theorem 3.1 is reduced to the following result.

Corollary 3.5. Let $C$ be a nonempty closed and convex subset of a Hilbert space $H$ and $\left\{T_{i}\right\}_{i \in I}: C \rightarrow$ $C$ a family of closed quasi-nonexpansive mappings such that $F=\bigcap_{i \in I} F\left(T_{i}\right) \neq \emptyset$. Let $\left\{\alpha_{n}\right\}$ be a real sequence in $(0,1)$ such that $\lim _{n \rightarrow \infty} \alpha_{n}=0$. Define a sequence $\left\{x_{n}\right\}$ in $C$ in the following manner:

$$
\begin{gathered}
x_{0} \in C \quad \text { chosen arbitrarily, } \\
y_{(n, i)}=\alpha_{n} x_{0}+\left(1-\alpha_{n}\right) T_{i} x_{n}, \\
C_{(n, i)}=\left\{z \in C:\left\|z-y_{(n, i)}\right\|^{2} \leq \alpha_{n}\left\|z-x_{0}\right\|^{2}+\left(1-\alpha_{n}\right)\left\|z-x_{n}\right\|^{2}\right\}, \\
C_{n}=\bigcap_{i \in I} C_{(n, i)}, \\
Q_{0}=C, \\
Q_{n}=\left\{z \in Q_{n-1}:\left\langle x_{n}-z, x_{0}-x_{n}\right\rangle \geq 0\right\}, \\
x_{n+1}=P_{C_{n} \cap Q_{n}} x_{0}, \quad \forall n \geq 0,
\end{gathered}
$$

then the sequence $\left\{x_{n}\right\}$ converges strongly to $P_{F} x_{0}$.

Remark 3.6. Corollary 3.5 includes the corresponding result of Martinez-Yanes and $\mathrm{Xu}$ [10] as a special case. To be more precise, Corollary 3.5 improves Theorem 3.1 of Martinez-Yanes and $\mathrm{Xu}$ [10] from a single mapping to a family of mappings and from nonexpansive mappings to quasi-nonexpansive mappings, respectively. 


\section{Acknowledgment}

This work was supported by the Korea Research Foundation Grant funded by the Korean Government (KRF-2008-313-C00050).

\section{References}

[1] F. E. Browder, "Fixed-point theorems for noncompact mappings in Hilbert space," Proceedings of the National Academy of Sciences of the United States of America, vol. 53, pp. 1272-1276, 1965.

[2] S. Reich, "Strong convergence theorems for resolvents of accretive operators in Banach spaces," Journal of Mathematical Analysis and Applications, vol. 75, no. 1, pp. 287-292, 1980.

[3] B. Halpern, "Fixed points of nonexpanding maps," Bulletin of the American Mathematical Society, vol. 73, pp. 957-961, 1967.

[4] P.-L. Lions, "Approximation de points fixes de contractions," Comptes Rendus de l'Académie des Sciences de Paris, Série. A-B, vol. 284, no. 21, pp. A1357-A1359, 1977.

[5] R. Wittmann, "Approximation of fixed points of nonexpansive mappings," Archiv der Mathematik, vol. 58, no. 5, pp. 486-491, 1992.

[6] N. Shioji and W. Takahashi, "Strong convergence of approximated sequences for nonexpansive mappings in Banach spaces," Proceedings of the American Mathematical Society, vol. 125, no. 12, pp. 3641-3645, 1997.

[7] H.-K. Xu, "Another control condition in an iterative method for nonexpansive mappings," Bulletin of the Australian Mathematical Society, vol. 65, no. 1, pp. 109-113, 2002.

[8] Y. Kimura and W. Takahashi, "On a hybrid method for a family of relatively nonexpansive mappings in a Banach space," Journal of Mathematical Analysis and Applications, vol. 357, no. 2, pp. 356-363, 2009.

[9] G. Lewicki and G. Marino, "On some algorithms in Banach spaces finding fixed points of nonlinear mappings," Nonlinear Analysis: Theory, Methods E Applications, vol. 71, no. 9, pp. 3964-3972, 2009.

[10] C. Martinez-Yanes and H.-K. Xu, "Strong convergence of the CQ method for fixed point iteration processes," Nonlinear Analysis: Theory, Methods \& Applications, vol. 64, no. 11, pp. 2400-2411, 2006.

[11] S.-Y. Matsushita and W. Takahashi, "A strong convergence theorem for relatively nonexpansive mappings in a Banach space," Journal of Approximation Theory, vol. 134, no. 2, pp. 257-266, 2005.

[12] S.-Y. Matsushita and W. Takahashi, "Weak and strong convergence theorems for relatively nonexpansive mappings in Banach spaces," Fixed Point Theory and Applications, vol. 2004, no. 1, pp. 37-47, 2004.

[13] K. Nakajo, K. Shimoji, and W. Takahashi, "Strong convergence by the hybrid method for families of mappings in Hilbert spaces," Nonlinear Analysis: Theory, Methods \& Applications, vol. 71, no. 1-2, pp. 112-119, 2009.

[14] K. Nakajo and W. Takahashi, "Strong convergence theorems for nonexpansive mappings and nonexpansive semigroups," Journal of Mathematical Analysis and Applications, vol. 279, no. 2, pp. 372$379,2003$.

[15] K. Nakajo, K. Shimoji, and W. Takahashi, "Strong convergence theorems by the hybrid method for families of mappings in Banach spaces," Nonlinear Analysis: Theory, Methods E Applications, vol. 71, no. 3-4, pp. 812-818, 2009.

[16] S. Plubtieng and K. Ungchittrakool, "Strong convergence theorems for a common fixed point of two relatively nonexpansive mappings in a Banach space," Journal of Approximation Theory, vol. 149, no. 2, pp. 103-115, 2007.

[17] X. Qin and Y. Su, "Strong convergence theorems for relatively nonexpansive mappings in a Banach space," Nonlinear Analysis: Theory, Methods E Applications, vol. 67, no. 6, pp. 1958-1965, 2007.

[18] X. Qin, Y. J. Cho, and S. M. Kang, "Convergence theorems of common elements for equilibrium problems and fixed point problems in Banach spaces," Journal of Computational and Applied Mathematics, vol. 225, no. 1, pp. 20-30, 2009.

[19] X. Qin, Y. J. Cho, S. M. Kang, and H. Y. Zhou, "Convergence of a modified Halpern-type iteration algorithm for quasi- $\phi$-nonexpansive mappings," Applied Mathematics Letters, vol. 22, no. 7, pp. 10511055, 2009.

[20] W. Takahashi, Y. Takeuchi, and R. Kubota, "Strong convergence theorems by hybrid methods for families of nonexpansive mappings in Hilbert spaces," Journal of Mathematical Analysis and Applications, vol. 341, no. 1, pp. 276-286, 2008. 
[21] W. Takahashi and K. Zembayashi, "Strong convergence theorem by a new hybrid method for equilibrium problems and relatively nonexpansive mappings," Fixed Point Theory and Applications, vol. 2008, Article ID 528476, 11 pages, 2008.

[22] W. Takahashi and K. Zembayashi, "Strong and weak convergence theorems for equilibrium problems and relatively nonexpansive mappings in Banach spaces," Nonlinear Analysis: Theory, Methods $\mathcal{E}$ Applications, vol. 70, no. 1, pp. 45-57, 2009.

[23] L. Wei, Y. J. Cho, and H. Y. Zhou, "A strong convergence theorem for common fixed points of two relatively nonexpansive mappings and its applications," Journal of Applied Mathematics and Computing, vol. 29, no. 1-2, pp. 95-103, 2009.

[24] H. Y. Zhou, G. Gao, and B. Tan, "Convergence theorems of a modified hybrid algorithm for a family of quasi- $\phi$-asymptotically nonexpansive mappings," Journal of Computational and Applied Mathematics, in press.

[25] Y. I. Alber, "Metric and generalized projection operators in Banach spaces: properties and applications," in Theory and Applications of Nonlinear Operators of Accretive and Monotone Type, A. G. Kartsatos, Ed., vol. 178 of Lecture Notes in Pure and Applied Mathematics, pp. 15-50, Marcel Dekker, New York, NY, USA, 1996.

[26] Y. I. Alber and S. Reich, "An iterative method for solving a class of nonlinear operator equations in Banach spaces," PanAmerican Mathematical Journal, vol. 4, no. 2, pp. 39-54, 1994.

[27] I. Cioranescu, Geometry of Banach Spaces, Duality Mappings and Nonlinear Problems, vol. 62 of Mathematics and Its Applications, Kluwer Academic, Dordrecht, The Netherlands, 1990.

[28] S. Kamimura and W. Takahashi, "Strong convergence of a proximal-type algorithm in a Banach space," SIAM Journal on Optimization, vol. 13, no. 3, pp. 938-945, 2002.

[29] W. Takahashi, Nonlinear Functional Analysis, Fixed Point Theory and Its Application, Yokohama Publishers, Yokohama, Japan, 2000.

[30] S. Reich, "A weak convergence theorem for the alternating method with Bregman distances," in Theory and Applications of Nonlinear Operators of Accretive and Monotone Type, A. G. Kartsatos, Ed., vol. 178 of Lecture Notes in Pure and Applied Mathematics, pp. 313-318, Dekker, New York, NY, USA, 1996.

[31] D. Butnariu, S. Reich, and A. J. Zaslavski, "Asymptotic behavior of relatively nonexpansive operators in Banach spaces," Journal of Applied Analysis, vol. 7, no. 2, pp. 151-174, 2001.

[32] D. Butnariu, S. Reich, and A. J. Zaslavski, "Weak convergence of orbits of nonlinear operators in reflexive Banach spaces," Numerical Functional Analysis and Optimization, vol. 24, no. 5-6, pp. 489-508, 2003. 